\title{
Elite Players' Perceptions of Football Playing Surfaces: A Qualitative Study
}

\author{
Jonathan R. Roberts*, Paul Osei-Owusu, Aimée C. Mears, and Andrew R. Harland
}

Sports Technology Institute, Wolfson School of Mechanical, Electrical and Manufacturing Engineering, Loughborough University, UK, LE11 3QF

KEYWORDS soccer, natural grass, artificial turf, injury.

\begin{abstract}
Purpose: The decision by the International Football Association Board in 2004 to approve the use of artificial surfaces in elite football (soccer) competitions remains controversial amongst many players, managers and coaching staff. The aim of this study was to conduct a comprehensive assessment of players' opinions to better understand the influence of playing surfaces on the game of football and identify factors that may contribute to differences of opinion. Method: Qualitative data were collected from 103 elite footballers and 21 coaching staff during a series of interviews and focus groups. A thematic analysis was conducted to identify patterns in the data. Results: Players considered that the type and condition of a playing surface influenced ball-surface interactions, game play, tactics/strategy, footwear selection, movement, risk of injury and fatigue. Together these influence a player's perception of the suitability of a surface and also their mindset, which could ultimately affect their performance. Conclusion: The majority of participants in this study expressed a higher preference for natural grass over artificial turf pitches. A perceived increased risk of injury on artificial turf remains a primary concern despite a lack of supporting evidence in research studies. To address this discrepancy, the reporting of muscle soreness and the effect of constant surface switching merit further consideration. Not all participants shared the same views and player characteristics such as age, surface experience, injury history and playing style/position were found to be potential factors that could account for differences in elite players' opinions regarding the surfaces used in football.
\end{abstract}

INTRODUCTION Football (or soccer) is a global game traditionally played on natural grass pitches. To thrive, grass needs a favorable environment in which to grow and, as a result, pitch quality can vary considerably (Caple, James, \& Bartlett, 2012). In 2004, third generation artificial turf was approved for use in competitive fixtures providing it meets a minimum set of requirements outlined in the FIFA Quality Programme (FIFA, 2015). The properties of an artificial pitch can also vary considerably due to construction method and wear (Alcántara, Gámez, Rosa, \& Sanchis, (2009); Sánchez-Sánchez, Felipe, Burillo, del Corral, \& Gallardo, L., 2014a; Sánchez-Sánchez et al., 2014b). An artificial pitch that has been certified by FIFA following a series of laboratory and field tests is referred to as Football Turf. Interestingly, no minimum requirements are in place for a natural grass pitch. The proposed benefits of Football Turf are that it is resistant to difficult climatic conditions, suitable for covered or steep-sided stadia which shade the pitch from sunlight, can be used for longer hours and for multiple uses (FIFA, 2015; FIFA, n.d.). At the elite level of the game, strong views regarding both natural grass pitches and artificial turf surfaces are frequently reported in the media especially when a player, coach or manager believes the surface has influenced or could influence the outcome of a game. Therefore, it is important to understand players' perceptions of the influence of the surface on the game of football.

A number of previous studies have attempted to analyze players' attitudes towards artificial turf. Using quantitative techniques primarily, Zanetti (2009) and Burillo, Gallardo, Felipe and Gallardo (2014) reported largely positive attitudes towards artificial turf whereas Johansson and Nilsson (2007) and Andersson, Ekblom and Krustrup (2008) found largely negative attitudes. These studies were conducted in different European countries, and the participants varied from amateurs to professional players, suggesting that attitudes could vary with ability level and between footballing populations from different countries. A range of possible factors that could explain differences of opinion have been reported in the literature including ability level (Burillo et al., 2014), gender (Andersson et al., 2008), playing position (Johansson \& Nilsson, 2007), age and surface experience (Burillo et al., 2014; Johansson \& Nilsson, 2007). Quantitative studies such as these provide appealing headline statistics but lack detailed explanations of the underlying reasons as opportunities to probe a participant's responses are limited when using quantitative techniques. 
Qualitative data, in comparison, provides greater depth and detail which can assist with understanding complex issues. Felipe et al. (2013) conducted a qualitative study to determine elite football players' requirements and perceptions of the advantages and disadvantages of artificial turf. The elite players in this study also generally expressed dissatisfaction with artificial turf and would not choose it as their preferred surface citing increased injury risk, abrasions from slide tackles and issues with ball-surface-players interactions as negative factors. Whilst this study provided many useful insights through the use of qualitative data, the sample was analyzed homogeneously and differences of opinion between players weren't considered. In addition, it focused solely on artificial turf.

The aim of this study was to conduct a comprehensive assessment of players' opinions to better understand the influence of playing surfaces on the game of football and identify factors that may contribute to differences of opinion. The focus of the study was on both artificial turf and natural grass rather than just artificial turf.

METHODS Four research questions were phrased to address the aim of this study:

RQ1 - What are the characteristics of a pitch deemed important by the players?

RQ2 - Which factors influence the condition of a pitch?

RQ3 - How do the characteristics of a pitch influence the way the game is played on a particular surface?

$\mathrm{RQ}_{4}$ - Are there differences of opinion between players and can these differences be related to a player's demographics, background or experience?

Participants Qualitative data were collected from 124 participants, most of whom (103) were footballers in the first team squad. The thoughts and opinions of members of staff were also deemed of interest and 21 coaches, physiotherapists and club doctors also participated in separate sessions to the players. The 103 elite footballers represented 23 different nationalities and contained 56 internationally capped players (senior, U-19, U-20, U-21). The mean age of the players was $25.7 \pm 4.2$ years. Further details on the participants in the study can be found in Table 1.

Purposeful sampling was employed in the selection of participants for the study. Seven clubs competing in either Ligue 1 in France or the Dutch Eredivisie (the top divisions in both countries), were visited during the second half of the 2010/2011 season. These two leagues were chosen as both divisions contained clubs that had installed a FIFA certified Football Turf pitch in their home stadium. These clubs were specifically approached to take part in the study as the players and staff would have experience of competing at a high level on a certified, modern artificial pitch.

Consequently, $51 \%$ of male players and $53 \%$ of their coaching staff who participated in this study were from four clubs (two in France and two in the Netherlands) that played their home games on an artificial pitch. In addition, further data was collected from players and coaches of the Netherlands women's national football team during a visit to their training camp.
Table 1. Detailed Breakdown of Participant Numbers by Role, Playing Position, Gender and Club League

\begin{tabular}{|c|c|c|c|c|c|c|c|}
\hline & \multicolumn{7}{|c|}{ Participant Numbers } \\
\hline Total & \multicolumn{7}{|c|}{124} \\
\hline Role & \multicolumn{3}{|c|}{$\begin{array}{c}\text { PLAYER } \\
103\end{array}$} & & \multicolumn{3}{|c|}{$\begin{array}{c}\text { STAFF } \\
21\end{array}$} \\
\hline Playing & GK & DEF & MID & FOR & \multicolumn{3}{|c|}{ - } \\
\hline Position & 13 & 32 & 39 & 19 & & & \\
\hline Gender & \multicolumn{2}{|c|}{86} & $\begin{array}{r}\text { FEM } \\
17\end{array}$ & ALE & \multicolumn{2}{|c|}{ MALE } & $\begin{array}{c}\text { FEMALE } \\
1\end{array}$ \\
\hline \multirow[t]{2}{*}{$\begin{array}{c}\text { Club } \\
\text { League }\end{array}$} & ERE & L1 & & . & ERE & L1 & - \\
\hline & 54 & 32 & & & 18 & 2 & \\
\hline
\end{tabular}

The following method was approved by the ethical committee at the authors' university and informed consent was obtained from all participants.

Data Collection Five investigators conducted semi-structured interviews and focus groups utilizing open-ended questions to generate qualitative data. This format was selected to give the participants the opportunity to express matters of significance to them rather than to obtain their opinion on matters deemed relevant by the investigators. Therefore, non-leading questions were used so that unanticipated responses could emerge and the direction of the discussion would be participantled. An interview guide was developed containing carefully constructed questions designed to initiate conversation with the participants; the guide however, provided sufficient freedom and flexibility to explore participant responses through the use of probes to seek clarification, elaboration or further detail. The use of an interview guide has proved successful in previous studies (Felipe et al., 2013; Roberts, Jones, Harwood, \& Mitchell, 20o1; Fleming, Young, Roberts, Jones, \& Dixon, 2005).

Thirty-two data collection sessions were conducted, the majority of which involved a focus group containing three to six participants led by one of the five investigators; the average session length was $30 \mathrm{~min} 44 \mathrm{~s}$ (standard deviation $7 \mathrm{~min} 41 \mathrm{~s}$ ). Focus groups were chosen as the dynamic nature of the discussion would enable different viewpoints to be raised, heard and discussed, which could highlight areas of agreement or disagreement (Patton, 2002). Data collection was conducted alongside a daily training session; availability of players and staff during the course of the session was primarily determined by the coach and, therefore, a degree of flexibility was required in terms of group sizes and interview locations. As a result, four individual interviews were also completed. The interviews and focus groups at Dutch clubs were conducted in English as the players and staff were able to speak the language to a very good standard. A translator was present during the visits to French clubs and during one session with the female Dutch players. Their role was to translate the interviewer's question, allow the discussion to evolve and then provide a brief summary to enable the interviewer to construct the next question. An audio recording of each session was 
captured then transcribed verbatim, following translation when necessary. For sessions involving a translator, only direct quotes from the players (translated into English) were used in the analysis, not the translator's summaries. Further data collection was deemed unnecessary as it was considered that a data saturation point had been reached and further sessions would be unlikely to yield any new information.

Data Processing and Analysis QSR-NVivo9 qualitative analysis software (QSR International 2010) was used to manage the large quantity of transcripts and organize the qualitative data. An analysis process was required that would enable the key themes to be identified without having to presuppose what they may be, thus enabling issues of importance to the participants to be ascertained. A thematic analysis was deemed appropriate for this study as it is a relatively straightforward method for identifying patterns in qualitative data that works well with relatively large data sets, can highlight similarities and differences as well as generate unanticipated insights (Braun and Clarke, 2006). The six main steps in the process as outlined by Braun and Clarke (2006) were followed: 1. familiarization with the data, 2. coding, 3. searching for themes, 4 . reviewing themes, 5. defining and naming themes, 6. reporting. The previously defined research questions were used to provide a degree of focus for the analysis. Multiple analysts were involved in the analysis process to identify and harmonize any different interpretations of the data.

RESULTS The results of the data analysis are presented in relation to the research questions outlined in the introduction. The characteristics of a pitch discussed by the players are briefly introduced before a more in-depth analysis of their perceived influence on the game of football is presented. Differences of opinion between players are then highlighted and where possible linked to the characteristics of the players.

\section{RQ1 - What are the characteristics of a pitch deemed} important by the players?

Perceived attributes of a pitch that players used to describe its condition or quality were grouped together in a general dimension entitled The Surface. The structure of sub-themes and example quotes associated with The Surface are illustrated in Figure 1. Important characteristics that were discussed include the quality and abrasiveness of the grass, the hardness and uniformity of the pitch, the grip and stability provided underfoot, surface moisture and smell.

\section{RQ2 - Which factors influence the condition of a pitch?}

The players highlighted a number of factors that they believed influenced the properties and condition of a playing surface. These quotes were grouped together and formed four further dimensions: Surface Type, Maintenance, Climate and Time of Year.
Influence of Climate and Time of Year: The study was conducted in Northern Europe, where environmental conditions vary considerably during the year due to climate. Players noted that the quality and hardness of natural grass is particularly susceptible to temperature, precipitation and sunshine and that the quality of a surface varies throughout the season. For many players, artificial turf is seen as being a solution to this problem as it can be played on all year round and in most weather conditions; although a number of players noted that the surface properties change with extremes of temperature.

Maintenance: The condition of a natural grass pitch was also linked to how well it is maintained particularly during the winter months. There was a general perception that artificial turf needs little maintenance but several people highlighted an issue with artificial grass fibers becoming flattened down over time.

Nobody knows how to do the maintaining, because it is new... natural grass we know all about it ... This is, for me, one of the problems that there still is. When they get the FIFA two star, it is tested all over, and it is the standard that it has to be. After one year it is a bit worse, but after two years and three years...it is getting harder and [there] is more slipping, I think that is the main problem. The grass is laying down it is not getting up again.

\section{RQ3 - How do the characteristics of a pitch influence the way the game is played on a particular surface?}

Quotes that related aspects of the game of football to the pitch attributes discussed in the previous section were grouped together in six dimensions: Footwear Selection, Movement, Injury and Fatigue, Tactics and Strategy, Game Play and Ball-Surface Interaction. Each dimension has a structure of sub-themes, however, a corresponding figure for each of these dimensions is not included due to space limitations.

Ball-Surface Interaction: Players were clear in their desire for the ball to go in the direction intended. Artificial turf was considered to have more predictable roll than natural grass due to the uniformity of the surface, although some artificial turf pitches were still criticized for being bumpy.

When you give a pass on the synthetic it will go [in a] straight line, but on grass it might surprise you, so the ball might go over your foot. [There are] no more surprises anymore when you play on synthetic because, if I pass the ball, I know where the ball will go, but on grass you don't know... it can be something in front of the goal, so you kick and the ball jumps over you, and that is the beauty of football.

The general consensus was that ball pace is faster on artificial turf compared with natural grass. 


\section{Sample Quotes}

Perfect pitch for me is a lot of grass, short and a bit of water on it. I think, the worst is not synthetic grass it is just a field with mud

It was more sand than grass

It was perfect, not too short, not too long the grass, a little bit wet. In Holland the last generations [of AT] are very good, because the length of the grass is a little bit longer and there is not too much rubber. Don't put too much rubber on, because when the grass is too short, it is not smooth anymore......

There's quite a lot of difference between [artificial pitches], because you have the very hard pitches and you also have the good ones.

It was like sand and really hard, no grass at all

Well you feel on the grass that it is firm and that you have a good balance on the grass...you feel free to move, change directions.

I don't like playing on artificial grass that is too hard. That's when I get problems. I like it when they are bit softer, not extremely soft. But soft.

It's softer; your feet can push into the ground, while on synthetic the feet cannot sink in

It was really bad. There was grass, but there were holes and bumps and, yes, it was a really bad pitch.

In one pitch different parts are different. There's a lot of grass, no grass, mud, or bumpy, so there are differences in one pitch

Artificial grass, the situation everywhere is the same, it is flat...

It was completely flat, the grass was perfect, perfect pitch.

I think every inch of the pitch has to be the same... like flat, it has to be firm to walk on.

...because [of] the instability of the pitch, so you have got a holes in the pitch, really soft underground. What you often see is the top surface and underneath there is a lot of sand, you know the building sand. And when you're turning you slide away completely..

And also... striking the ball, the support leading into a strike isn't at all the same as on grass.... I find you don't get the same support.

As a keeper, when I play on grass, I use studs. This gives better support.

it's just so hard and you don't have the same grip as you have on grass as you get really low. Here it is like Bambi on ice, it is terrible, The pitch changes a lot and is probably more slippery in the winter time than some of the natural ones... in the winter [it is] very dangerous, because it is icy and when it is wet it is really slippery

You get too deep into the ground, and you can say that I am having a lot of grip, but you cannot move.

They have got more grip on artificial than they have on grass

When it is wet and you move to the right or left or sometimes you slide with your feet, and when it is dry you get more grip. But the grip is still enough to move left or right..., so then it is no problem.

It was perfect like a green, short. But also they put water on it before the game, it was really really nice.

On an artificial you will always have the same pitch, the only difference you can make is if it is wet or dry.

I believe you always have to put water on it before playing

Before, the old synthetic pitches, it was like a rough rug that would burn you, it was really hard... the first synthetics were really challenging... when you tackled someone you could burn your thighs but now it's softer, it's more like a grass field.

if it is warm in the summer then the rubber smells

I also think in the summer, when the sun is on the grass the smell, I think it is going to smell
Base Themes

Sub-Themes

Dimension

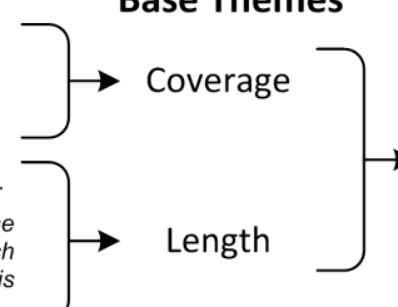

Grass Quality

Hard

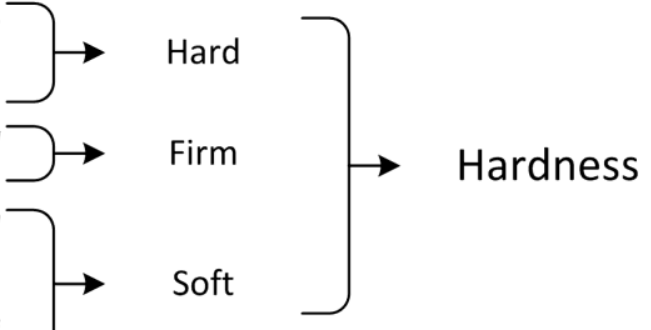

$\longrightarrow$ Regular
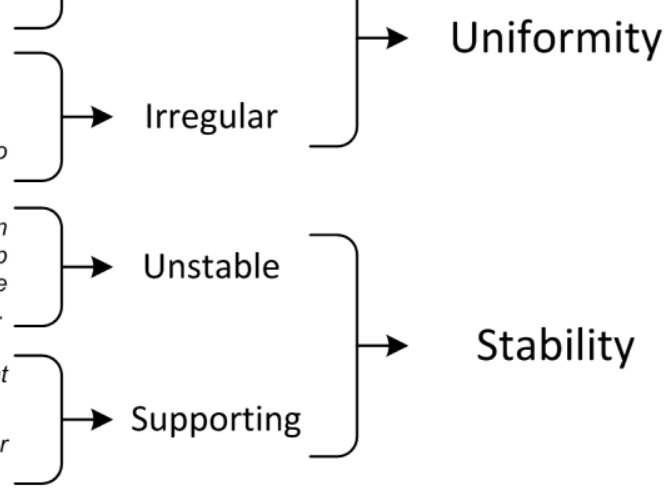

Supporting

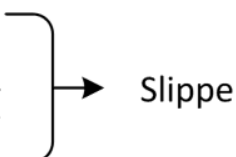

$\longrightarrow$

More Grip

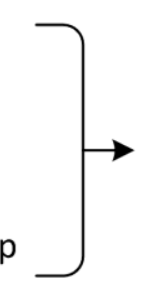

\section{Grip}

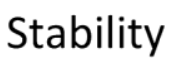

The Surface

\section{Surface \\ Moisture}

\section{Abrasiveness}

Smell

Figure 1. "The surface" dimension showing sub-structure generated from quotes. 
It affects the way we play... the strength we can put into the ball when passing... judging [a] pass accurately is completely different from a natural grass pitch... the ball can get away very fast, when on a grass pitch the ball is held back some more, it "brakes" it a little bit more.

Ball pace/roll was linked to grass length and, for both natural grass and artificial turf, problems were reported with either too long or too short grass.

Surface moisture was considered to have a big influence on ball interactions with both natural grass and artificial turf, affecting ball pace, rolling and sliding. Players were particularly critical of dry artificial turf pitches which were described as too slow; wet pitches were preferred but then the pace was perceived to be very fast, faster than natural grass, '[Artificial turf] does not have a middle, the ball is very fast or very slow.'

Ball bounce was also widely perceived to differ between natural grass and artificial turf but few players were able to describe differences clearly. Players also noted that ball bounce is affected by surface moisture, perceiving the ball to skid through when wet and sit up when dry, an effect that can be exaggerated on an artificial pitch.

Surface moisture can be a result of either manual pitch irrigation or precipitation. Players overwhelmingly preferred a pitch to be watered before a game but complained about an inconsistent approach especially for artificial pitches. Even if they are watered, in warm, sunny weather the pitch dries out and players have to adapt their style of play. Surface wetness due to the weather can have a big influence on the pitch as it is uncontrollable.

Game Play: Quotes relating to skills, tasks or actions performed during the course of a game of football that were perceived to be influenced by the surface were grouped together within Game Play. On an uneven surface, players indicated that they need to concentrate more, as ball control is more difficult, more touches may be needed which gives the opponent an opportunity to put them under pressure.

When the ball is bumping... it is so difficult. You have to concentrate fully on your first touch and when the field is flat and good, you can concentrate on more things.....

Players believed that a flat uniform pitch encourages a passing game because the ball goes where the player intends, fewer touches are needed and, therefore, one-touch football can be played. The uniformity of an artificial pitch is, therefore, considered to encourage a passing game involving short passes along the ground.

Players indicated that the tempo of a game is typically higher on artificial turf as the surface pace is usually faster so the ball can be moved around quicker. Passes, however, need to be more precise on a fast paced pitch or the ball can get away from a player and possession is lost. Players, therefore, avoid longer passes, and strikers in particular noted that longer passes are more difficult on artificial turf because the ball bounces away.

We don't specifically have instructions, but the fact of playing on synthetic means we play shorter balls. We avoid long passes. It's the pitch that requires a different way of playing.

Defenders, however, also, indicated that they had to adjust their game to suit an artificial pitch, having to anticipate the game better because the tempo is faster and the ball interacts differently. In addition, defenders described being more reluctant to slide tackle on artificial turf, preferring to stay on their feet to avoid skin burns, although there was an acknowledgement that sliding is less painful if the pitch is wet. As a result, players consider a match on artificial turf to be less physical with less contact between players.

When you come out on a pitch and you see grass, you see a little bit of wetness on the grass you feel happy. Me, I am defender so I feel finally I can make a sliding [tackle], but when I see the synthetic, I am thinking, "my skin burns, please my skin..." so I am holding myself [back].

Tactics and Strategy: Players and coaches described how different styles of play are suited to different surfaces primarily because of the effect of surface conditions on ball interactions. Passing teams need a good quality surface and so uneven pitches were considered to give weaker teams a better chance because they hinder the more skillful, technical, passing teams. Some coaches, therefore, adjusted their tactics as a result of the playing surface opting for a more direct approach on poor quality pitches.

The pitch was so terrible that the coaches said that we don't want to take any risk, we played the long ball...We said at the beginning, watch out because if you play short passes it is dangerous, as the ball bounces, don't take any risk, just play the long ball.

Although players and coaches acknowledge that certain styles of play are more suited to artificial turf (e.g. passing game) there seems to be little attempt made to change a team's style of play when changing from natural to artificial pitches. The biggest consideration for coaches in terms of their strategy appeared to be in preparation for a game. Teams that play their home matches on artificial pitches typically trained all week on the pitch before a home game and all week on a natural turf pitch before an away game. Other teams predominantly trained on natural grass and train for a couple of days, at the most, on artificial turf in preparation for an away game on football turf. Because of the variability from pitch-to-pitch (for both natural grass and artificial turf pitches) some coaches questioned the benefit in trying to prepare on a matching surface. 
Before the Heracles game, we trained the whole week on artificial turf, but if it is not the same artificial turf and there are not a lot of these surfaces in Holland. If it is not the same, even training the whole week on artificial turf does not have any advantage.

The players and staff reported concerns that if they train for longer on artificial turf the players will be injured by the time of the game. Some coaches indicated that they tone down the training on football turf or build up the intensity slowly to minimize the risk of injury, whilst others prepared in the same way, the content of the training doesn't change. Other coaches have decided it is not worth the risk of injury at all and have ceased to prepare on artificial turf.

It is not worth going into the game and feeling already stiffness in your muscles, because you trained on the synthetic. So we only train on grass.

Coaches also reported adjusting their training on a natural turf pitch due to the condition of the surface.

Two weeks ago, ...the field we were training on had lots of rain, so the pitch was soft... I am sure that the legs of the players they get tired, because they are not used to playing on that pitch. It was soft so I cut down the training session...

Movement: Players strongly linked their ability to move effectively with the hardness and grip of a pitch. Natural grass pitches were acknowledged to have variable hardness; low levels of grip were predominantly linked with very hard pitches, although firm pitches that don't cut up were considered desirable. Players expressed differing views on the grip provided by artificial pitches compared to natural grass which may be linked with varying perceptions of the hardness of synthetic turf. Older pitches in particular were considered harder perhaps due to degradation with age. Players generally felt they had more difficulty twisting and turning on artificial turf, which wasn't solely related to a lack of grip. There was a feeling that their studs get 'stuck' in the ground and it takes them longer to turn. Relatively few players thought they could turn quickly on football turf.

I have the feeling on artificial you get stuck a lot you don't have the freedom in the ankles or knees to move, because you don't feel comfortable turning and twisting.

It may be that players experience high levels of grip but, perhaps fearing an injury, adapt the way they move to limit the risk; this, however, results in complaints that they cannot stop or change direction as well.
The stops are different. Stopping is a lot more complicated. To come to a stop after a quick run, to block and start again, it's a lot more difficult on synthetic.

You have to take a lot of small steps, actually, before being able to block. While on grass, you can come to a full stop and start again. Here it's not the case. We tend to be carried further by our momentum.

Players felt more comfortable on natural turf because the soil gives when braking and so movements are more natural. If the ground is too soft and unstable, however, then players described having little grip, increasing the risk of injury.

On normal grass, your shoe will turn like this on the grass, but on artificial it sticks, it does not turn... you have to take your foot out if you want to turn... on real grass you can keep your foot on the ground and then turn.

Generally players felt they could run faster on artificial turf, unless it is a very soft pitch. In a number of these themes, players have linked how they move on different surfaces and the level of grip with injury potential, which is discussed further in the next section.

Injury and Fatigue: Players perceived there to be a much higher risk of getting injured on artificial turf compared to natural grass.

When you talk about injuries, it can happen anywhere, but the risk is higher on a synthetic, because you can just make a small direction change, it can happen that your knees are gone, [or] your Achilles.... It can also happen on a normal pitch but the chances [of it] happening are minimal.

A variety of different injury types and locations were associated with artificial turf including knee and ankle joints and back pain. Perhaps the most common injury type discussed was muscle or joint soreness. Many of the injuries were referred to as 'aches' or 'pains' that didn't appear to require longer term treatment. The perceived hardness of an artificial pitch was a major factor highlighted by numerous players whilst the intensity of a competitive match and the faster tempo of the game on artificial surfaces were also thought to contribute.

When I got used to artificial grass I could tell the difference between normal grass and artificial. I started to feel the pain, because sometimes the ball runs so fast on artificial, you have to run faster, sometimes when you do that you hurt yourself, but you don't know it then because of the tempo of the game you just want to run after the ball.

Usually you feel more pain after a match on synthetic; pain in the back and joints, because a match, it's more 
intense. During training we take more breaks, we have time to recover... while during a match you don't have time to think, you have to be $100 \%$ focused for one and a half hours, and often the physical problems occur after a match.

Many players complained about skin burns when falling or sliding on artificial turf, particularly when it is very hot or dry. This had implications for performing slide tackles. Players complained about it being painful on the hips when falling, particularly goalkeepers when diving. Goalkeepers also reported similar problems with elbows.

When you come in, you see a synthetic, the first thing in your mind is no, my back, my knee. Because you don't want to go onto the field and make a sliding tackle because you know that you are going to get skin burns, it's a pain... but on natural grass you will love it, then you know that there is nothing going to happen to you.

Typically players perceived a soft natural grass pitch to be tiring to play on; this seemed particularly noticeable for those that play regularly on artificial turf. Mixed views were expressed on whether artificial turf is more or less tiring than natural grass and views again appeared to be related to the surface type a player was accustomed to using.

I think it is dependent on how much you play on artificial and how much you play on grass, because we play every day on artificial, when we play on grass my legs are more tired after a game, because I am not used to it.

There was a general feeling that regular switching between natural grass and artificial turf causes more injury problems than constantly playing on one surface type. This issue with switching surfaces was often linked to the differences in the hardness between pitches.

Grass, because of its flexibility, I think it reduces forces in joints. On an artificial pitch it is mostly a bit harder and that is what they feel... when they play repetitively on that kind of court there is no problem anymore, because the body will adapt to that, but if they change a lot then they say ok we feel it.

The previous section described how players linked surface stability and grip with their ability to move effectively. Consequently, players were concerned that twisting/turning or changing direction on artificial turf can lead to injury. There was however, no clear understanding of any relationship between footwear and injury. Some players believed there to be a link others suggested not. Footwear selection is discussed further in the following section.

Footwear Selection: Players described how they choose their footwear to suit the type and condition of a pitch. Most players stated that they wear shorter length molded studs on artificial turf. A few indicated that they use small studs but generally they are considered too long and unstable. Differences of opinion existed between players regarding the use of blades on artificial turf. A number of players declared that they use blades although at least one club involved in the study advises against their use. A handful of players use shoes specifically designed for artificial turf.

There are a lot of players who play on synthetic grass pitch with little studs, a lot of little studs, there are also guys with normal studs or blades... It is difficult because there is not one line to follow.

On natural turf most players stated that they wear screwin studs. Occasionally molded boots are used if it is firm or dry but very few used blades. A few players stated that they use the same boots regardless of the pitch. It was found that choice of boot is largely the player's decision. At some clubs, staff indicated that they try to advise but found that players tend to want to wear the boots they are used to playing in or use their sponsor's boot.

There is no medical reason for us as medical staff to advise that they have to wear different shoes, so we leave that decision to themselves.

Players also acknowledged the influence of environmental and surface conditions on boot selection, opting for longer or screw-in studs to improve grip and maintain the ability to move effectively when it is wet, slippery or soft underfoot. The goalkeepers involved in the study indicated they use long studs regardless of the playing surface, even on artificial turf.

Adaption: The previous sections have highlighted the differences that players perceive between pitches. Differences between natural grass and artificial turf became the focus of many discussions but the players also acknowledged that natural grass differs from pitch to pitch, as does artificial turf.

...you have to adapt to the pitch, you cannot expect to have a perfect pitch every time you play, that is why you go and look at the pitch before the game and then you see ok what am I going to expect today.

The concept of adapting to the condition of the surface was discussed in relation to each of the six dimensions Footwear Selection, Movement, Injury and Fatigue, Tactics and Strategy, Game Play and Ball-Surface Interactions. Views differed considerably on how long it takes to adapt from 15 minutes up to a month.

I think that when you're a top sportsman then you do not need two days to experience the grass. When we play at 2:30 and we do the warming up at 2:0o, then my body is used [to it] after 15 minutes on the pitch... your body adapts very fast. I told my colleagues ... go blind to the 
game do your adaption during the warming up and play 90 minutes.

Well, for us, in order to really get some good results, it almost took us a month.

A number of players believed that the time taken to adapt to an artificial surface gave teams that play their home games on synthetic pitches an advantage, however, a player from one such club made the following remark.

We are swapping pitches every weekend, so for them it is only two times a year, for us it is every week we change, so we have to adapt to that.

$\mathrm{RQ}_{4}$ - Are there differences of opinion between players and can these be related to a player's demographics, background or experience?

The Player: There was some evidence in the data that the characteristics and previous experiences of a player could influence their attitudes, perceptions or preferences towards different surfaces or their ability to perform on different surfaces. Characteristics of a player that were linked to a playing surface included their mindset, physical attributes (e.g. age), playing style and position. Factors such as surface experience and injury history that could influence a player's perceptions were grouped together in separate dimensions.

There was some evidence that preconceived ideas about artificial pitches based on previous surface experiences could affect a player's mindset.

The first generation artificial fields was where they played hockey, and in the winter when there was snow on the pitch we always trained on those kind of pitches but they were hard, if you dropped down. And this was what was in in our head.

A number of players admitted that having a poor mindset towards an artificial surface could affect their performance.

When you see $100 \%$ artificial grass, there is no way your brain is going to focus and you want to play football. Because you're more afraid of getting injuries rather than going to play football

I think if you step on a field which you think is a bad field, you will get injured, you will feel pain, you will feel every pain, instead of you saying when you step on the field... ok I am going to play soccer.

There was an indication that older players tended to be more critical of artificial pitches than younger players, who have a more positive mindset. There is a perception that young players can adapt but older players are more at risk of injury. Concern was also expressed that playing regularly on artificial turf may shorten careers.

In the youth your muscles are always flexible, in youth it's better to play on artificial grass. You always have a good pitch for the youth to play on, your muscles are always good to play. Later on when I turned 18, when you switch all the time between artificial and grass, I feel my knees and everything. When I became older it's more difficult a pitch to play on because it is harder.

There was a suggestion that artificial pitches are more suited to short, quick, nimble players or intelligent, skillful, technical players. As a result they may have a more positive mindset about playing on artificial pitches than those players that may struggle to adapt.

If you have a person who is, let's say, technically comfortable will be more likely to choose synthetic than a person who isn't technically comfortable, who struggles on synthetic.

... you need a certain technical quality to be able to evolve on synthetic. When you are technically good, it's easier to adapt.

As discussed in the section on Game Play, surface conditions can affect players in different positions. Some players suggested that artificial turf benefits attacking players due to the fast pace of the pitch encouraging high tempo passing whilst the reluctance to slide tackle was felt to hamper defenders, although not all agreed. Goalkeepers indicated that they dislike diving on artificial turf as it is hard, usually dry, burns and they get rubber infill in their faces. Players in different positions may therefore have different attitudes to artificial pitches depending on the perceived benefits of different surfaces for their game.

For every goalkeeper, I think it is a disaster for your hips... for your elbow, you can never go $100 \%$ on this pitch.

The Player - Contributing Factors: Although there was limited evidence, there was a suggestion that previous experience of an injury may influence a player's mindset towards artificial pitches.

Some players they don't even play on the artificial grass because they are scared of injuries. You see it a lot. Some players, they got injured really bad once ...[now] they don't play, they say sorry coach.

When I heard that Excelsior wanted to have me here on loan, at first I thought that I had to play on Astro turf, I think not so good for my ankle, because I had an operation on it. Up to now it is going ok because this is soft Astro turf 
During the interviews, players were asked to describe their experiences of the different types of pitches that they had played on during their life.

I grew up also playing on clay. I hear the children in Sweden now, when I read the newspapers, they complain about artificial grass. Yeah that's a bit worrying I must say. When we grew up we played on clay. I hated it so much during the winter and now the children are complaining about bad artificial pitches. I don't understand it. They are spoilt I think.

A complete, comprehensive record of surface experience was not achieved to enable a detailed comparison with differences in attitudes and opinions although a number of general trends were apparent. Surface experience appeared to be related to the perceived risk of injury on an artificial pitch and fatigue on different types of pitch (as discussed in Section 3.2.5). The majority of players that didn't consider artificial turf to be a major source of injury problems were notably based at clubs with artificial turf as their home pitch.

Everybody says in the beginning that with artificial grass the chance for injury is much higher, but I play now for 8 years and... not many injuries this season not last season.

Relationship Model Relationship models or maps have been used previously to represent findings in an easyto-understand, visual manner (Roberts et al., 2001; Felipe et al., 2013). Little distinction, however, has been made between very different dimensions which may represent physical attributes of a product, performance aspects, perceived benefits or outcomes, characteristics of the athlete, socio-psychological factors etc. The relationship model in Figure 2 attempts to overcome these issues and illustrates the latest development of an initial concept outlined by Ronkainen, Osei-Owusu, Webster, Harland and Roberts in 2012. The Surface is the physical entity of primary concern for this study and the important attributes that players used to define the condition of the surface are subthemes of this dimension. Comparison with Figure 1 illustrates how the tree structure for The Surface has been represented in the relationship map. Factors that are perceived to affect the condition of a surface are then illustrated feeding in.

The six aspects of the game that players' believed were influenced by surface properties and condition are presented as central dimensions all linked to The Surface with the concept of 'adaption' enclosing and encompassing all six dimensions. The sub-themes for each of these dimensions are again shown around the periphery and links between the central dimensions illustrate, for example, how the players described that footwear selection influences movement which can affect injury risk and therefore lead to different strategies.

The Player is presented as a second physical entity, defined by a series of characteristics that form the sub- themes for this dimension. Again contributing factors that may influence a player's opinion are illustrated. The Player is linked to each of the central dimensions to illustrate that the characteristics and experiences of a player may influence their perceptions of the game of football on different surfaces. The model enables further studies focusing on other physical entities such as the ball and the boot to expand the map in future.

DISCUSSION AND CONCLUSION The perceived advantages and disadvantages of natural grass and artificial turf have been documented previously to a greater or lesser extent in a number of publications. In this study, players expressed an overwhelming preference for natural grass pitches over artificial turf. Both Andersson et al. (2008) and Felipe et al. (2013) found negative attitudes towards artificial turf amongst elite footballers in Europe. Felipe et al. (2013) found that players would not choose artificial turf as their habitual surface but still went on to conclude that "most of the players and coaches consider that artificial turf is now ready to be introduced to first-class European football and that they would not mind playing matches on this surface regularly". In addition they considered that "professional football's inhibitions regarding artificial turf may very possibly soon be reduced to a minimum". The latter statement was based on an assumption that, because of the widespread usage of artificial turf at youth level, the next generation of players will be more accustomed to artificial pitches and, therefore, be happier to use it. This study has also found that younger players may be more positive towards the use of artificial turf (as did Burillo et al., 2014), perhaps because they have only experienced the latest generation of artificial pitches or because their youthful bodies are more able to adapt, however, the danger with Felipe et al.'s assumption is that attitudes may well change with time. Older players believed that, because of their age, they were more likely to sustain an injury on artificial turf. If younger players experience more injuries that they associate with an artificial surface as they grow older then their attitudes may well change. In addition, there is the unknown influence of other players in the dressing room; as youth players graduate to the senior squad they may become influenced by the attitudes and opinions of dominant experienced figures who, at this time, are typically critical of artificial turf.

The perceived increased risk of sustaining an injury was a major factor contributing to negative attitudes to artificial pitches. The concerns of the players, however, do not correspond with the findings of research studies, which have generally found little if any difference in injury rates between natural and artificial turf (Williams, Hume, Kara, 2011). Perhaps the most common injury type discussed was muscle or joint soreness that didn't necessarily put a player out of action. Soreness has been highlighted as a problem for players in previous injury perception studies (Poulos et al., 2014) yet reporting of soreness in studies of injury prevalence may not always be achieved as it may not satisfy the definition of an injury used in those studies. 


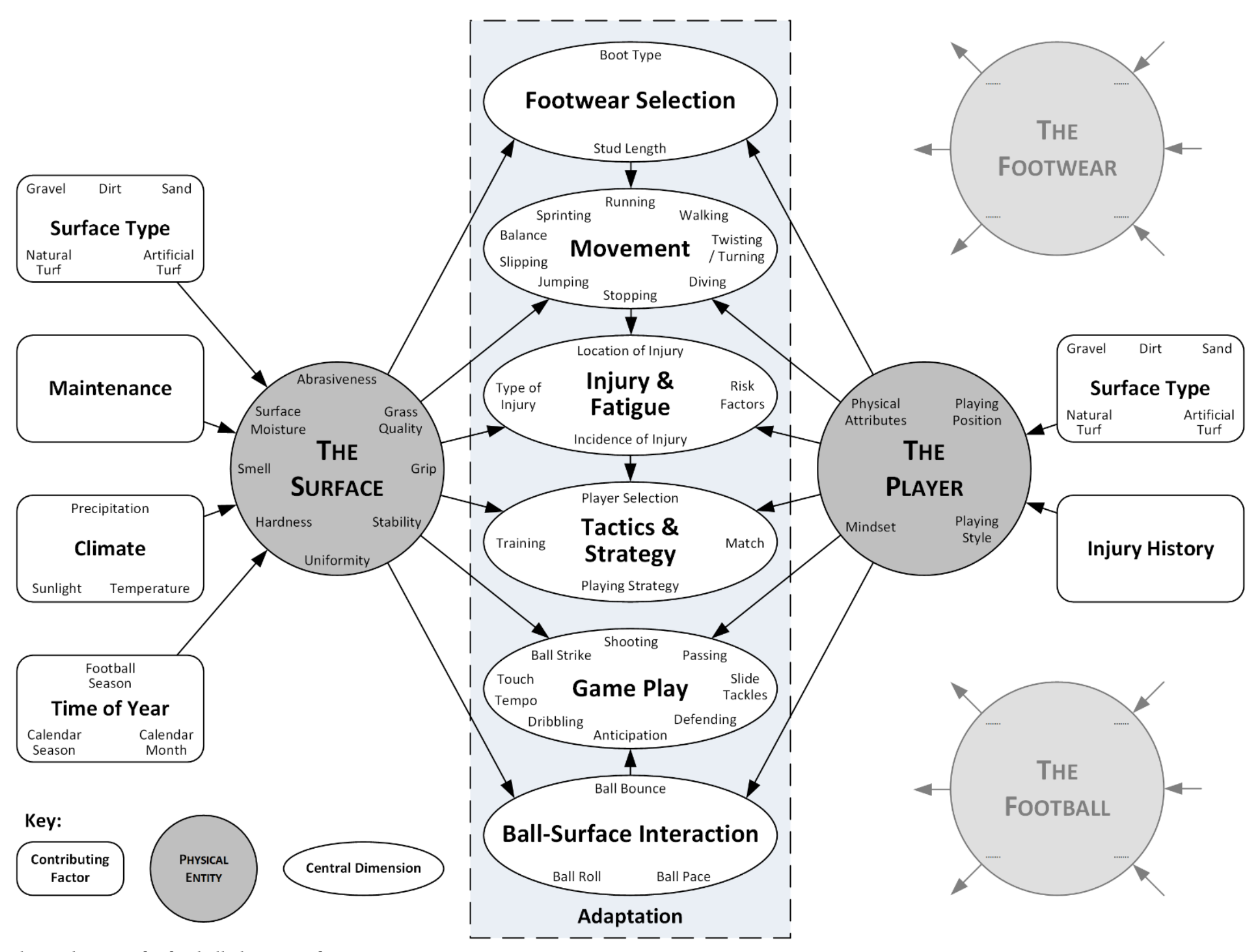

Figure 2. Relationship map for football playing surfaces 
Nédélec et al. (2013) found that players reported moderately higher soreness in a small selection of muscles over a period of 48 hours after conducting a 90 minute soccer specific field test on artificial turf compared to natural grass. No differences in physical measures of fatigue were found, however, leading them to conclude that exercising on artificial turf doesn't result in greater fatigue or delayed recovery. The authors did acknowledge that the subjects were young and familiar with playing on artificial surfaces, and this study suggests that this demographic will be more adaptable to artificial pitches.

Constant switching between different surface types during a season was identified by players and staff as a possible risk factor for feelings of soreness and fatigue, which is consistent with a study by Williams et al. (2011). A more recent investigation found no link found between surface shifting and time loss to overuse injuries for Scandinavian players (Kristenson et al., 2016); players from Scandinavian countries such as Sweden and Iceland, however, have more artificial turf experience compared to many football nations which could have contributed to the results as they are more accustomed to artificial turf. Furthermore, it is difficult to determine whether soreness was captured as an injury in the study by Kristenson et al. (2016) which may have led to different conclusions regarding surface switching.

In contrast to the negative attitudes reported, Burillo et al., (2014) found that $\sim 75 \%$ of participants were highly satisfied with artificial pitches and Zanetti (2009) reported higher ratings for artificial turf amongst their data sets. Burillo et al. (2014) also reported that overall satisfaction with artificial surfaces reduced with increases in ability level and surface experience on natural turf. Both these studies focused on amateur or semi-pro players whose experiences of natural surfaces were of dirt pitches rather than pristine grass. It is perhaps not surprising therefore that an artificial turf pitch would be desirable in comparison. With increasing ability level, players tend to be provided with better quality natural grass pitches and for those competing in the highest divisions and the major tournaments, expectations change as a result. Players in this study considered there to be sufficient financial resource at the top level and ample evidence that high quality natural turf pitches can be maintained throughout the year despite adverse weather conditions in Northern Europe and, therefore, see little reason for clubs to use artificial turf. Surface experience and expectations are therefore likely to be the underlying factors that explain changes in attitude with ability level.

Although many players were strongly in favor of natural grass, a number acknowledged that they would rather play on artificial turf than poor quality natural grass, favoring artificial turf in the winter months but returning to natural grass during the warmer, drier, sunnier months of the year when natural grass pitches are easier to prepare. Interestingly, in this study there was emerging evidence that, at clubs which use an artificial pitch for their home matches, the players have adapted to the surface over time and some, though certainly not all, expressed more positive attitudes towards artificial turf particularly with regard to injury. No such trend, however, was evident in the data collected by Andersson et al. (2008). In this study, previous experiences of different surfaces appeared to have a significant influence on the mindset of a player, with negative attitudes towards a surface potentially influencing their performance. This study also revealed that the demands of different playing positions could lead to different attitudes between goalkeepers, defenders and attackers. Zanetti (2009) also noted differences in preference with playing position although the trend wasn't clear.

Players frequently discussed the hardness of different surfaces which they found to vary considerably due to the type of pitch, environmental conditions and the maintenance of aging surfaces. Risk of injury and their ability to move effectively on a surface were specifically linked to the hardness of a pitch. Extremes of hardness (both too soft and too hard) were criticized, although a number of players advocated a firm surface. Sánchez-Sánchez et al. (2014b) found that sprint performance improved and that players reported feeling the most comfortable on the hardest artificial surface they tested, although the players weren't exposed to long enough durations of testing on each surface to perhaps experience any negative consequences of the hardest pitch. Nevertheless, it suggests that the hardness of pitch needs to be finely balanced between optimizing performance and minimizing complaints particularly about the perceived risk of injury.

Players and coaches expressed concerns over the way artificial turf ages and is maintained. Artificial turf is typically viewed as being cheaper and easier to maintain than grass. Players however were very critical of older pitches where the fibers had begun to lay down and were harder underfoot. Manufacturers of artificial turf face a difficult challenge in balancing the desire to have soft fibers and low slip resistance for lower abrasion with the need for fibers to remain resilient and upright to provide resistance to ball roll (Fleming, 2011). Whilst generic guidance for maintenance has been introduced into the FIFA Quality Programme, Fleming (2011) has proposed that the intensity of use and subtle differences in the surface system design demand site-specific maintenance programs.

The open-ended, qualitative approach used in this study has provided detailed, in-depth understanding of players' attitudes to football playing surfaces. Attempts have been made to identify consensus between players or highlight contradictions in the data. By the very nature of this approach, however, there can be a lack of comparability between interviews and, as a result, valuable insights may be highlighted and discussed by only a few participants. In addition, the study contained a relatively small sample of players from Northern Europe making it difficult to generalize the findings. Finally the study was conducted in $2010 / 11$ and it is possible that attitudes will change over time.

What does this article add? The use of artificial pitches at the elite level of the game remains controversial and continues to be resisted by football players. The decision to hold the 2015 Women's World Cup was met with protests and legal action, whilst a 2018 consultation 
amongst clubs in the Dutch Eredivisie has resulted in restrictions on new installations and incentives for clubs to revert back to natural turf. Player dissatisfaction however is not always consistent with research findings. This paper presents the most comprehensive study to date capturing the thoughts, perceptions and opinions of over 100 players and coaches regarding the different surfaces used in football. It has sought to build on previous work by providing enhanced understanding of the effect of surface properties on the game of football and, particularly, by identifying characteristics and experiences of a player that may influence their perceptions of playing surface suitability. Rather than treating players as a homogeneous group, future research should consider factors such as the player's age, playing position, playing style, surface experience, injury history, their expectations and mindset. Injury risk remains a major issue perceived by the players particularly regarding artificial turf; this study has identified potential reasons for the disconnect between players' perceptions of injury risk and research into injury rates on different surfaces. Future research should consider muscle soreness which might not be adequately reported when using clinical definitions of injury. In addition, switching between surfaces was perceived to be a potential risk factor which has received little research attention.

\section{AUTHOR INFORMATION}

\section{Corresponding Author}

* Jonathan R Roberts; L.R.Roberts@lboro.ac.uk. Sports Technology Institute, Loughborough University, 1 Oakwood Drive, Loughborough LE11 3QF, UK

\section{Author Contributions}

The manuscript was written through contributions of all authors.

\section{Funding Sources}

This work was supported by the Fédération Internationale de Football Association (FIFA).

\section{ACKNOWLEDGMENT}

The authors wish to thank FIFA for funding this research project as well as FIFPro, the Member Associations and players for their input and help to achieve the objectives of this study. The authors would also like to thank Jouni Ronkainen and James Webster for their assistance with the data collection.

\section{REFERENCES}

Alcántara, E., Gámez, J., Rosa, D., \& Sanchis, M. (2009). Analysis of the influence of rubber infill morphology on the mechanical performance of artificial turf surfaces for soccer. Proceedings of the IMechE, Part P: Journal of Sports Engineering and Technology, 223(1), 1-9.

Andersson, H., Ekblom, B., \& Krustrup, P. (2008). Elite football on artificial turf versus natural grass: movement patterns, technical standards, and player impressions. Journal of Sports Sciences, 26(2), 113-122.

Braun, V. \& Clarke, V. (2006). Using thematic analysis in psychology. Qualitative Research in Psychology, 3(2), 77-101.
Burillo, P., Gallardo, L., Felipe, J. L., \& Gallardo, A. M. (2014). Artificial turf surfaces: perception of safety, sporting feature, satisfaction and preference of football users. European Journal of Sport Science, 14 (Suppl 1), S437-447.

Caple, M., James, I., \& Bartlett, M. (2012). Mechanical behaviour of natural turf sports pitches across a season. Sports Engineering, 15(3), 129-141.

Felipe, J. L., Gallardo, L., Burillo, P., Gallardo, A., Sánchez-Sánchez, J., \& Plaza-Carmona, M. (2013). A qualitative vision of artificial turf football fields: elite players and coaches. South African Journal for Research in Sport, Physical Education and Recreation, 35(2), 105-120.

FIFA (2015). FIFA Quality Programme for Football Turf: Handbook of Requirements (October 2015 Edition). Retrieved from https://football-technology.fifa.com/media/1239/fqp-handbook-of-requirements-v26.pdf

FIFA (n.d.). About Football Turf. Retrieved from https://football-technology.fifa.com/en/media-tiles/about-football-turf/

Fleming, P. (2011). Maintenance best practice and recent research. Proceedings of the IMechE, Part P: Journal of Sports Engineering and Technology, 225(3), 159-170.

Fleming, P. R., Young, C., Roberts, J. R., Jones, R., \& Dixon, N. (2005). Human perceptions of artificial surfaces for field hockey. Sports Engineering, 8(3), 121-136.

Johansson, F. \& Nilsson, P. (2007). Swedish football players and artificial turf - a study about their attitudes regarding artificial turf and related injuries. Retrieved from http://www.spelarforeningen.com/wp-content/uploads/2009/10/English_version_sfs_study_200707.pdf

Kristenson, K., Bjørneboe, J., Waldén, M., Ekstrand, J., Andersen, T. E., \& Hägglund, M. (2016). No association between surface shifts and time-loss overuse injury risk in male professional football. Journal of Science and Medicine in Sport, 19(3), 218-221.

Nédélec, M., McCall, A., Carling, C., Le Gall, F., Berthoin, S., \& Dupont, G. (2013). Physical performance and subjective ratings after a soccer-specific exercise simulation: Comparison of natural grass versus artificial turf. Journal of Sports Sciences, 31(5), 529-536.

Patton, M. Q. (2002). Qualitative Research and Evaluation Methods (3rd ed.). London, UK: Sage.

Poulos, C. C., Gallucci, J., Gage, W. H., Baker, J., Buitrago, S., \& Macpherson, A. K. (2014). The perceptions of professional soccer players on the risk of injury from competition and training on natural grass and 3rd generation artificial turf. BMC Sports Science, Medicine and Rehabilitation, 6(1), 11-17.

Roberts, J., Jones, R., Harwood, C. \& Mitchell, S., (2001). Human perceptions of sports equipment under playing conditions. Journal of Sports Sciences, 19, 485-497.

Ronkainen, J., Osei-Owusu, P., Webster, J., Harland, A., \& Roberts, J. (2012). Elite player assessment of playing surfaces for football. Procedia Engineering, 34, 837-842.

Sánchez-Sánchez, J., Felipe, J. L., Burillo, P., del Corral, J., \& Gallardo, L. (2014a). Effect of the structural components of support on the loss of mechanical properties of football fields of artificial turf. Proceedings of the IMechE, Part P: Journal of Sports Engineering and Technology, 228(3), 155-164.

Sánchez-Sánchez, J., García-Unanue, J., Jiménez-Reyes, P., Gallardo, A., Burillo, P., Felipe, J. L., \& Gallardo, L. (2014b). Influence of the mechanical properties of third-generation artificial turf systems on soccer players' physiological and physical performance and their perceptions. PloS One, 9(10), e111368. 
Williams, S., Hume, P., \& Kara, S. (2011). A review of football injuries on third and fourth generation artificial turfs compared with natural turf. Sports Medicine, 41(11), 903-923.
Zanetti, E. M. (2009). Amateur football game on artificial turf: players' perceptions. Applied Ergonomics, 40(3), 485490. 